\title{
Sleep better on combination therapy: SLFN11 predicts response to veliparib and temozolomide in recurrent small cell lung cancer
}

\author{
Chen Zhao, Nobuyuki Takahashi, Arun Rajan \\ Thoracic and Gastrointestinal Malignancies Branch, Center for Cancer Research, National Cancer Institute, National Institutes of Health, Bethesda, \\ MD, USA \\ Correspondence to: Arun Rajan, MD. Thoracic and Gastrointestinal Malignancies Branch, National Cancer Institute, National Institutes of Health, \\ 10 Center Drive, 10-CRC, Room 4-5330, Bethesda, MD, USA. Email: rajana@mail.nih.gov. \\ Comment on: Pietanza MC, Waqar SN, Krug LM, et al. Randomized, Double-Blind, Phase II Study of Temozolomide in Combination With Either \\ Veliparib or Placebo in Patients With Relapsed-Sensitive or Refractory Small-Cell Lung Cancer. J Clin Oncol 2018;36:2386-94.
}

Submitted Dec 09, 2018. Accepted for publication Dec 14, 2018.

doi: $10.21037 /$ tlcr.2018.12.11

View this article at: http://dx.doi.org/10.21037/tlcr.2018.12.11

Small cell lung cancer (SCLC) is a devastating disease with rapid progression and limited treatment options (1). Topotecan is the only Food and Drug Administration (FDA) approved treatment option for recurrent or progressive SCLC, but has a median time to progression of less than four months (2). More effective therapies are urgently needed. Alkylating agents have shown single-agent efficacy in SCLC (3), and a recent phase II clinical trial demonstrated that temozolomide (TMZ), a non-classic oral alkylating agent, had activity in relapsed SCLC [overall response rate (ORR) 20\%], particularly for brain metastasis (4). However, the median progression-free survival (PFS) time of TMZ was still only 3.5 months.

Poly (ADP-ribose) polymerase (PARP)-dependent base excision repair is a well-defined mechanism of resistance to TMZ (5). Adding veliparib, an inhibitor of PARP1 and PARP-2, to TMZ can overcome TMZ resistance in preclinical models (6). A proteomic profiling study discovered elevated expression level of PARP-1 in SCLC (7). Additionally, aberrant expression of several genes associated with DNA damage response is observed in SCLC (8). Hence, PARP inhibitors are attractive candidates for targeting DNA damage response pathways in SCLC (9).

Pietanza $e t$ al. have conducted a multicenter, randomized, double-blind, phase II study (ClinicalTrials.gov identifier: NCT01638546) to test the hypothesis that adding veliparib to TMZ may overcome resistance and improve outcome in patients with relapsed SCLC (10). A total of 104 patients with recurrent SCLC were enrolled and randomly assigned 1:1 to receive oral veliparib (40 $\mathrm{mg}$ twice daily, from day 1 to 7 ) or placebo with TMZ (150 to $200 \mathrm{mg} / \mathrm{m}^{2} /$ day, day 1 to 5 of a 28-day cycle) as second- or third-line therapy until disease progression, unacceptable toxicity, or withdrawal of consent. Response to treatment was determined by imaging at weeks 4 and 8 , and every 8 weeks thereafter. The primary endpoint was 4-month PFS. Although TMZ/ veliparib showed a higher ORR (39\% vs. 14\%; $\mathrm{P}=0.016)$, 4-month PFS and overall survival (OS) was not significantly different between the two groups: $36 \%$ versus $27 \%(\mathrm{P}=0.19)$, and 8.2 versus 7.0 months $(\mathrm{P}=0.50)$, respectively. The combination of TMZ with veliparib was associated with a higher incidence of neutropenia and thrombocytopenia. These observations are similar to the experience of combining PARP inhibitors with cytotoxic chemotherapies (11). A reduction in the dose of TMZ from 200 to $150 \mathrm{mg} / \mathrm{m}^{2} /$ day resulted in a significant reduction in the incidence of hematological toxicities. The investigators concluded that more frequent myelosuppression, treatment delays, dose reduction and a higher ratio of patients with platinumresistance in the TMZ/veliparib group may have contributed to the discordance between higher ORR and no improvement in survival. Although the primary endpoint was not achieved, subgroup analysis showed that patients with Schlafen-11 (SLFN11) expression, an exploratory endpoint of this clinical trial, had a significantly better PFS (5.7 vs. 3.6 months; $\mathrm{P}=0.009)$ and $\mathrm{OS}(12.2$ vs. 7.5 months; $\mathrm{P}=0.014)$ with TMZ/ veliparib treatment.

SLFN11 is a putative DNA/RNA helicase belonging to the Schlafen family of proteins which cause cell cycle arrest (from the German word Schlafen: "to sleep") (12). 
It functions as a cell cycle checkpoint and induces lethal $\mathrm{S}$-phase arrest in response to DNA damage (13). Two groups independently discovered that SLFN 11 expression predicts sensitivity to DNA damaging agents (DDA) based on screening of the Cancer Cell Line Encyclopedia (CCLE) (14) and the National Cancer Institute Antitumor Cell Line Panel (the NCI-60) (15). Preclinical studies also confirm that SLFN11 expression can predict sensitivity to PARP inhibitors by trapping PARP1 and PARP2 at DNA singlestrand breaks ("PARP trapping") $(16,17)$. Patient-derived xenograft (PDX) models have demonstrated that SLFN11 expression measured by immunohistochemistry (IHC) correlates with treatment responses to PARP inhibitors in SCLC (17).

Pietanza and colleagues amended their study to investigate if SLFN11 expression was predictive of clinical benefit from TMZ/veliparib and showed that SLFN11 is a predictive biomarker for both PFS and OS in SCLC patients receiving PARP inhibitors and TMZ (10). Interestingly, there was a trend to improved OS calculated from initial diagnosis in SLFN11-overexpressing SCLCs, which raises the question whether SLFN11 overexpression identifies SCLC patients destined to do better regardless of the type of DDAs administered rather than identifying a subset of SCLC patients who might derive greater benefit from PARP inhibitors in combination with DDAs. Nevertheless, the results reported by Pietanza and colleagues warrants further investigation in a prospective study that stratifies SCLC patients to receive PARP inhibitors and TMZ based on their SLFN11 expression level.

Although associated with a strong biological rationale, the addition of veliparib to TMZ does not improve survival in an unselected group of SCLC patients and comes at a price in terms of toxicity. Therefore, it is important to identify variables associated with the combination which can be optimized to maximize clinical benefit. SLFN11 overexpression is one such variable as mentioned above.

The choice of PARP inhibitor and continuous drug dosing by minimizing dose interruptions can also potentially influence clinical activity (18). The most potent PARP inhibitors generally demonstrate the strongest DNA binding. Talazoparib is one of the most potent PARP inhibitors with greater PARP trapping potency than veliparib and a strong correlation of cytotoxic potential with SLFN11 expression (16). To minimize dose interruptions caused by hematological toxicities, dose modification of one or both members of the combination can be considered. Recent preclinical data suggests that lower doses of TMZ can be combined with maximum doses of PARP inhibitors without compromising clinical activity, while reducing dosing interruptions due to hematological toxicities (19). These data provide a rationale for combining talazoparib with sub-maximal doses of TMZ in future studies of SCLC.

In addition to SLFN11 expression, attempts are underway to develop biomarkers, such as RAD51 and $\gamma \mathrm{H} 2 \mathrm{AX}$, that can identify patients most likely to benefit from PARP inhibitors $(8,18)$. Gene expression signatures have been developed to predict sensitivity to PARP inhibitors and identify synergistic drug combinations (20). PARP-targeted imaging is a novel approach that permits assessment of target engagement in vivo and can help study the effects of variations in dose and dosing regimens (21).

Pietanza and colleagues have shown that targeting DNA damage pathways with a combination of a PARP inhibitor and an alkylating agent can improve clinical outcome and survival in patients with SLFN11-expressing SCLCs. Other mechanisms to target DNA damage and repair pathways also need to be investigated, especially for SLFN11deficient tumors. Mitotic inhibitors such as aurora kinase and cell cycle checkpoint inhibitors are currently under investigation (8). Ataxia telangiectasia mutated (ATM) and ATM- and Rad3-related (ATR) kinases exert a regulatory effect on DNA damage and repair pathways (22). The ATR inhibitor, M6620 is active in combination with topotecan in patients with platinum-refractory SCLC and is currently under investigation in an ongoing phase II clinical trial (NCT02487095) (23).

Based on the observations of Pietanza and colleagues, we propose a rational design for future clinical trials for patients with platinum-refractory SCLC (Figure 1). For tumors with high SLFN11 expression, a combination of an alkylating agent with a PARP inhibitor warrants further evaluation. For SLFN11-deficient tumors, other treatment options should be explored. Epigenetic modifications such as methylation, acetylation and EZH2 mediated H3K27me deposition can influence SLFN11 expression. Drugs targeting these epigenetic modifiers, such as DNA demethylating agents (decitabine), histone deacetylase (HDAC) inhibitors (romidepsin, entinostat) and EZH2 inhibitors (EPZ011989), have been shown to successfully reverse SLFN11 deficiency-associated resistance to conventional chemotherapy and DDA in preclinical models and can be evaluated in clinical trials (24). The HDAC inhibitor, belinostat in combination with cisplatin and etoposide has shown clinical activity in patients with SCLC and neuroendocrine tumors in a phase I clinical trial (25). 


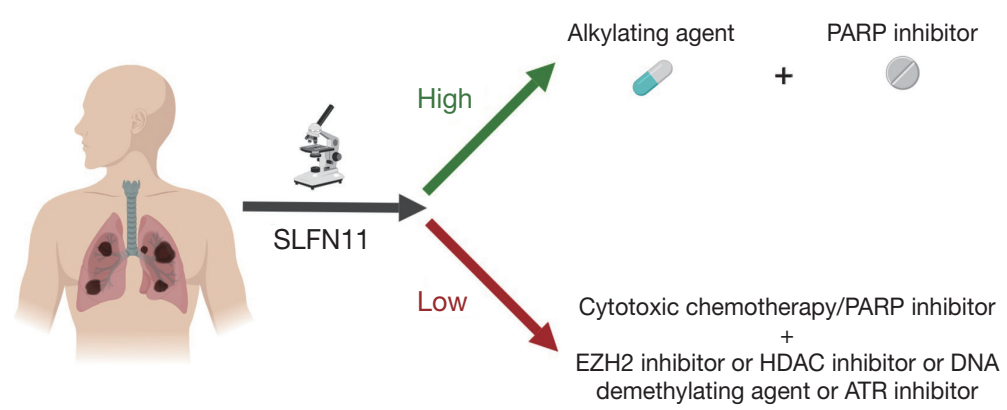

Figure 1 Possible treatment strategy for SCLC based on SLFN11 expression. Patients with high SLFN11 expression level (H score $\geq 1$ ) could potentially benefit from combination of an alkylating agent and a PARP inhibitor. For patients with SLFN11-deficient tumors, adding EZH2 inhibitors, HDAC inhibitors, DNA demethylating agents or ATR inhibitors to cytotoxic chemotherapy or PARP inhibitors can potentially overcome resistance to DNA damaging therapies caused by SLFN11 deficiency. SCLC, small cell lung cancer; PARP, Poly (ADP-ribose) polymerase; HDAC, histone deacetylase. (This figure was created with BioRender.)

ATR inhibition has also been shown to overcome resistance to PARP inhibitors in SLFN11-deficient tumors, providing a rationale for combining ATR inhibitors with PARP inhibitors in patients with SLFN11-deficient tumors (16). These approaches might also find applicability in other SLFN11deficient cancers such as colon cancer and ovarian cancer (13).

\section{Acknowledgements}

Funding: This research was supported in part by the Intramural Research Program of the NIH, National Cancer Institute, Center for Cancer Research.

\section{Footnote}

Conflicts of Interest: The authors have no conflicts of interest to declare.

\section{References}

1. Farago AF, Keane FK. Current standards for clinical management of small cell lung cancer. Transl Lung Cancer Res 2018;7:69-79.

2. O'Brien ME, Ciuleanu TE, Tsekov H, et al. Phase III trial comparing supportive care alone with supportive care with oral topotecan in patients with relapsed small-cell lung cancer. J Clin Oncol 2006;24:5441-7.

3. Green RA, Humphrey E, Close H, et al. Alkylating agents in bronchogenic carcinoma. Am J Med 1969;46:516-25.

4. Pietanza MC, Kadota K, Huberman K, et al. Phase II trial of temozolomide in patients with relapsed sensitive or refractory small cell lung cancer, with assessment of
methylguanine-DNA methyltransferase as a potential biomarker. Clin Cancer Res 2012;18:1138-45.

5. Tentori L, Graziani G. Chemopotentiation by PARP inhibitors in cancer therapy. Pharmacol Res 2005;52:25-33.

6. Palma JP, Wang YC, Rodriguez LE, et al. ABT-888 confers broad in vivo activity in combination with temozolomide in diverse tumors. Clin Cancer Res 2009;15:7277-90.

7. Byers LA, Wang J, Nilsson MB, et al. Proteomic profiling identifies dysregulated pathways in small cell lung cancer and novel therapeutic targets including PARP1. Cancer Discov 2012;2:798-811.

8. Foy V, Schenk MW, Baker K, et al. Targeting DNA damage in SCLC. Lung Cancer 2017;114:12-22.

9. Pietanza MC, Byers LA, Minna JD, et al. Small cell lung cancer: will recent progress lead to improved outcomes? Clin Cancer Res 2015;21:2244-55.

10. Pietanza MC, Waqar SN, Krug LM, et al. Randomized, Double-Blind, Phase II Study of Temozolomide in Combination With Either Veliparib or Placebo in Patients With Relapsed-Sensitive or Refractory Small-Cell Lung Cancer. J Clin Oncol 2018;36:2386-94.

11. Rajan A, Carter CA, Kelly RJ, et al. A phase I combination study of olaparib with cisplatin and gemcitabine in adults with solid tumors. Clin Cancer Res 2012;18:2344-51.

12. Schwarz DA, Katayama CD, Hedrick SM. Schlafen, a New Family of Growth Regulatory Genes that Affect Thymocyte Development. Immunity 1998;9:657-68.

13. Ballestrero A, Bedognetti D, Ferraioli D, et al. Report on the first SLFN11 monothematic workshop: from function to role as a biomarker in cancer. J Transl Med 2017;15:199.

14. Barretina J, Caponigro G, Stransky N, et al. The Cancer Cell Line Encyclopedia enables predictive modelling of 
anticancer drug sensitivity. Nature 2012;483:603-7.

15. Zoppoli G, Regairaz M, Leo E, et al. Putative DNA/ RNA helicase Schlafen-11 (SLFN11) sensitizes cancer cells to DNA-damaging agents. Proc Natl Acad Sci U S A 2012;109:15030-5.

16. Murai J, Feng Y, Yu GK, et al. Resistance to PARP inhibitors by SLFN11 inactivation can be overcome by ATR inhibition. Oncotarget 2016;7:76534-50.

17. Lok BH, Gardner EE, Schneeberger VE, et al. PARP Inhibitor Activity Correlates with SLFN11 Expression and Demonstrates Synergy with Temozolomide in Small Cell Lung Cancer. Clin Cancer Res 2017;23:523-35.

18. Brown JS, Kaye SB, Yap TA. PARP inhibitors: the race is on. Br J Cancer 2016;114:713-5.

19. Smith MA, Reynolds CP, Kang MH, et al. Synergistic activity of PARP inhibition by talazoparib (BMN 673) with temozolomide in pediatric cancer models in the pediatric preclinical testing program. Clin Cancer Res 2015;21:819-32.

20. McGrail DJ, Lin CC, Garnett J, et al. Improved prediction of PARP inhibitor response and identification of

Cite this article as: Zhao C, Takahashi N, Rajan A. Sleep better on combination therapy: SLFN11 predicts response to veliparib and temozolomide in recurrent small cell lung cancer. Transl Lung Cancer Res 2018;7(Suppl 4):S308-S311. doi: 10.21037/tlcr.2018.12.11 synergizing agents through use of a novel gene expression signature generation algorithm. NPJ Syst Biol Appl 2017;3:8.

21. Carney B, Kossatz S, Lok BH, et al. Target engagement imaging of PARP inhibitors in small-cell lung cancer. Nat Commun 2018;9:176.

22. Zeman MK, Cimprich KA. Causes and consequences of replication stress. Nat Cell Biol 2014;16:2-9.

23. Thomas A, Redon CE, Sciuto L, et al. Phase I Study of ATR Inhibitor M6620 in Combination With Topotecan in Patients With Advanced Solid Tumors. J Clin Oncol 2018;36:1594-602.

24. Gardner EE, Lok BH, Schneeberger VE, et al. Chemosensitive Relapse in Small Cell Lung Cancer Proceeds through an EZH2-SLFN11 Axis. Cancer Cell 2017;31:286-99.

25. Balasubramaniam S, Redon CE, Peer CJ, et al. Phase I trial of belinostat with cisplatin and etoposide in advanced solid tumors, with a focus on neuroendocrine and small cell cancers of the lung. Anticancer Drugs 2018;29:457-65. 